\title{
Personalised Learning Spaces and Federated Online Labs for STEM Education at School
}

\author{
Supporting Teacher Communities and Inquiry Learning
}

\author{
Denis Gillet \\ EPFL \\ Lausanne, Switzerland \\ denis.gillet@epfl.ch
}

\author{
Ton de Jong \\ University of Twente \\ Enschede, The Netherlands \\ a.j.m.dejong@utwente.nl
}

\author{
Sofoklis Sotirou \\ Ellinogermaniki Agogi \\ Athens, Greece \\ sotiriou@ea.gr
}

\author{
Christophe Salzmann \\ EPFL \\ Lausanne, Switzerland \\ christophe.salzmann@epfl.ch
}

\begin{abstract}
The European Commission is funding a large-scale research project on federated online laboratories (Labs) for education in Science, Technology, Engineering, and Mathematics (STEM) at School. The main educational focus is on inquiry learning and the main technological one is on personalized learning spaces. The learning spaces are offered through a single European social media portal supporting simultaneously teacher communities and student learning activities. This paper presents the general technical framework devised to support the construction and the exploitation of learning spaces, as well as the federation of online labs.
\end{abstract}

Keywords-Online Labs; Remote Labs; Virtual Labs; Inquiry Learning; Learning Spaces; Personalization; Federated Repositories; Teacher Communities; Social Media; Communities of Practice

\section{INTRODUCTION}

The European Commission is funding a large-scale research project on federated online laboratories (Labs) for education in Science, Technology, Engineering, and Mathematics (STEM) at School called Go-Lab. The Go-Lab project opens up remote science laboratories, their data archives, and virtual models referred to hereafter as online labs for large-scale use in education. Go-Lab enables science inquiry-based learning that promotes acquisition of deep conceptual domain knowledge and inquiry skills and directs students to careers in science. For students (10 to 18-years old), Go-lab offers the opportunity to perform personalized scientific experiments with online labs in pedagogically structured and scaffolded learning spaces that are extended with social communication facilities. For teachers, Go-Lab offers pedagogical "plug, share, and play" through a Webbased interface and a community framework to disseminate best practices and find mutual support. A modular approach and inquiry classroom scenarios promote a seamless incorporation of online labs into the classroom. For lab owners, Go-Lab provides open interfacing solutions to easily plug in their online labs, construct virtual didactic components, and share them in the Go-Lab federation of online labs.

The first two sections of this paper present the current challenges that have impaired the spreading of online labs in education and the solutions proposed to make the federation and the exploitation of online labs simple. Then, the concept of online inquiry learning spaces is developed, with a focus on personalization and collaboration through a dedicated social media portal for STEM at school. The social media portal is based on the Graasp ${ }^{1}$ platform developed in the framework of the Palette (2006-2009) and ROLE ${ }^{2}$ (2009-2013) European projects dedicated to the participatory design and validation of online support for communities of practice and personal learning environments (PLE), respectively.

\section{CHALLENGES IN ONLINE LABS}

Nowadays, access to remote or virtual laboratory facilities is provided in blended learning or distance learning frameworks for schools and universities. These remote labs are offered by large scientific or pedagogically oriented organizations, as well as by universities or individual providers. The labs offered differ widely in domain, intention, interface, and learner support. The diversity of the accessible online labs is, of course, a great advantage, as teachers may exploit them in their lessons to cover many topics. This diversity, however, also partly form the foundation for a number of barriers that prevent teachers from adopting remote labs as learning resources. These barriers are:

- Existing online labs usually have no structuring and scaffolding for the inquiry process.

- Existing online labs differ in interface and usage possibilities, which makes them less usable in the classroom.

- Existing online labs are not geared towards a specific age group and therefore often do not fit.

- Existing online labs are not organized along domains (rather on topics), which makes that teachers cannot integrate more online labs over a longer period.

- For existing online labs it is often unclear where they fit into STEM curricula.

$$
\begin{gathered}
{ }^{1} \mathrm{http} / / / \text { graasp.epfl.ch } \\
\text { htth://wwww:role-project.edt }
\end{gathered}
$$


- Most STEM teachers are not aware of online lab technologies and hence do not grasp their benefits.

- Teachers are not sufficiently trained in using online labs: they rarely implement activities in class unless they feel confident with the process and can troubleshoot problems.

- There is no support for teachers from the online lab owners.

- There is no community of teachers who use online labs.

- There are no tools or support to easily customize, localized or repurpose online labs for alternative scenarios or different contexts.

- ICT infrastructure in schools may not be sufficient for use of online labs, e.g., difficulty to book computer laboratory time, low bandwidth Internet access.

- Existing online labs may not implement the high level of security required when dealing with anonymous or untrained user access.

- Existing online labs often required browser plug-ins that may not be up to date and can only be updated by system administrators in schools.

These barriers force teachers, if they use online labs, to only exploit them on an incidental base. They can be overcome by offering structure and scaffolding for experimentation with online labs, by providing students with a standardized interface, by making online labs adaptable to context (age, discipline, language), by organizing online labs following "big ideas", by indicating where online labs (and the associated activities) fit into a curriculum, by setting up extensive awareness building activities, by providing teachers with dedicated support facilities, by connecting schools and lab owners, by creating and strengthening online teacher communities, and by organizing dedicated support infrastructures and events.

\section{TECHNICAL FRAMEWORK FOR ONLINE LABS FEDERATION AND EXPLOITATION}

To tackle most of the challenges mentioned above and to ease deployment at school, a social media Portal is proposed for STEM education (Green boxes in Fig. 1). This portal is designed as a single European gateway to online labs, associated inquiry learning activities [1], peer learners and teacher communities. All the necessary end-user personalization features and inquiry learning apps integrated in the Portal are designed and will be implemented in three phases including validation at school to take into account preferences, competence levels, subject domains, and contexts, without intervention of administrators or developers [2].

The Go-Lab Portal will be the single entry point for all stakeholders integrating individual labs or repositories, learning activity spaces, associated resources and supporting communities. It will include simple interfaces, search and recommendation, as well as aggregation and scaffolding features available in contextual spaces created, shared and repurposes by the teachers themselves. Go-Lab will rely on the Go-Lab backend infrastructure (Black boxes in Fig. 1) to support Lab owners in providing and sharing their resources, as well as to support teachers, communities and students in enriching, sharing and exploiting these resources. The associated centralized data sets will enable to construct learning analytics and provide personalized and contextualized awareness, scaffolding, recommendation, and customization while enforcing privacy.

The infrastructure designed to support the federation and exploitation of online labs [3] with the Portal relies on four main backend services. The Lab owner services allow the easy plug-in of online labs by large scientific organizations or universities. The Cloud Services offer a federation of online labs allowing their sharing, while Learning Analytics and Scaffolding Services provide facilities the Portal relies on. Addon services enable the integration of additional features for booking and bartering.

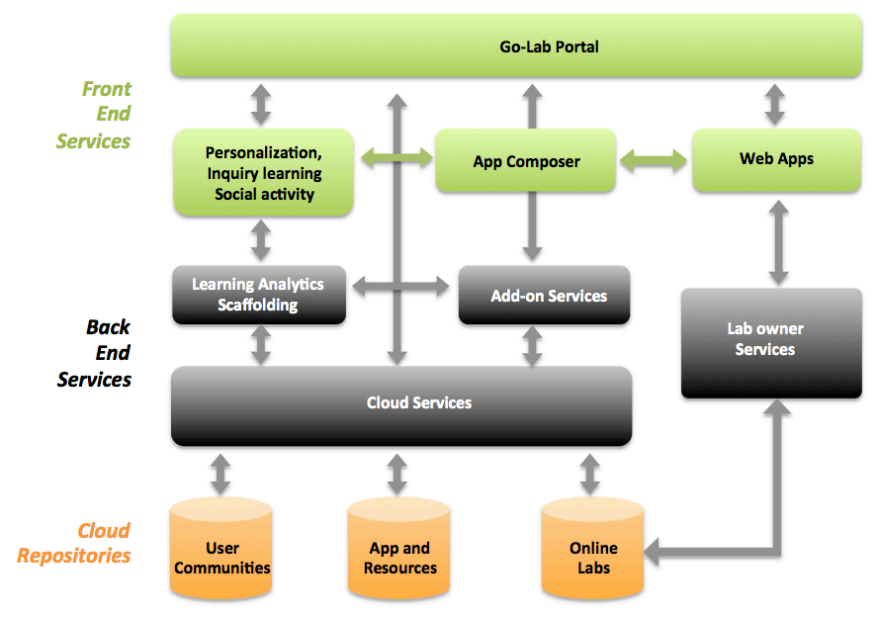

Figure 1. The simplified view of the Go-Lab technical infrastructure.

One of the technological challenges of the Go-Lab project is to enable the connection (Plug) to all the envisioned online labs using a standardized interface. While the Web apps implemented as Opensocial gadget permits standardization at the user interface level, the backend standardization will rely on the smart device paradigm [4] to enable the connection to existing or new online labs.

The advent of Opensocial gadgets or widgets combined with the recent evolution of browser standards (HTML5, CSS, Javascript) enable the design of Web applications (apps) that match features of similar desktop applications. These technologies enable an easy design and aggregation of apps with the help of the App Composer, as well as the integration of additional services within the Portal.

One key technology upon which gadgets relies on is websocket ${ }^{3}$. It enables the fine control of the communication channel at the browser level, which is essential to exchange data with online labs. Streaming large amount of data or video becomes feasible using websocket if the available bandwidth is sufficient and efficiently controlled [5].

\footnotetext{
${ }^{3}$ http://tools.ietf.org/html/rfc6455
} 
The smart device paradigm revisits the traditional clientserver approach by re-engineering server side to integrate more intelligence (Fig. 2). This will make the remote device more ubiquitous, more autonomous, and more self-aware of it surrounding. For example, the smart device may look for other smart devices in its subnet. It may query them about their availability, capabilities and be ready to reply to such peer requests by returning among other information a mathematical model of the experiment and a Virtual 3D Model. It may even provide model parameters that were autonomously identified. With this information, it is possible to fully simulate the experiment. The simulation can also be combined with the information acquired on the physical experiment to provide an augmented view of the online lab [6].

The smart device should be aware of its own state and detect via auto diagnostic if there is a malfunction. If such problems occur, it should be able to send alarms or diagnostic reports.

The smart device should support all types of connections and protocols present or future. While expecting such support is a daydream, carefully designing the structure and the services offered by the smart device will permit an easier integration with the Cloud Services and the Add-on services and also simplify updates and evolution that will occur in the life cycle of the smart device.

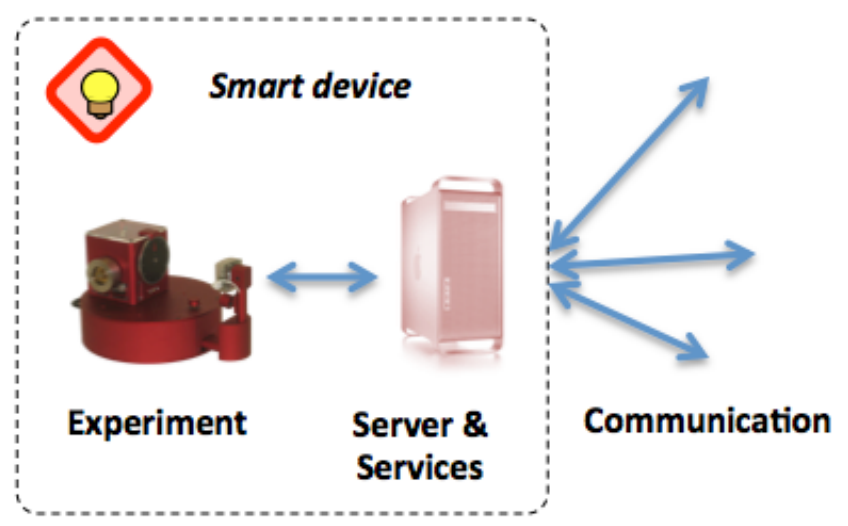

Figure 2. The Smart Device paradigm places additional intelligence at the server side to provide more services and ubiquitous interaction.

For existing online labs that do not yet have the infrastructure or capabilities to connect to existing services, the adjunction of a smart gateway will extend the existing features and permit an effortless integration (Fig. 3). The advantage of such gateway is that the existing online labs will require minimal or no change to be integrated in the Cloud. For example important existing features like remote device security [7] -think about controlling a remote telescope- will not be handled by the smart gateway but by existing security procedures. On the other hand, adding a gateway comes with the drawback that real-time communications may be delayed, which could be an issue for some labs.

The lab owners shall be provided with a service that will allow the easy plug of their labs online while the cloud services will provide the federation between these labs.
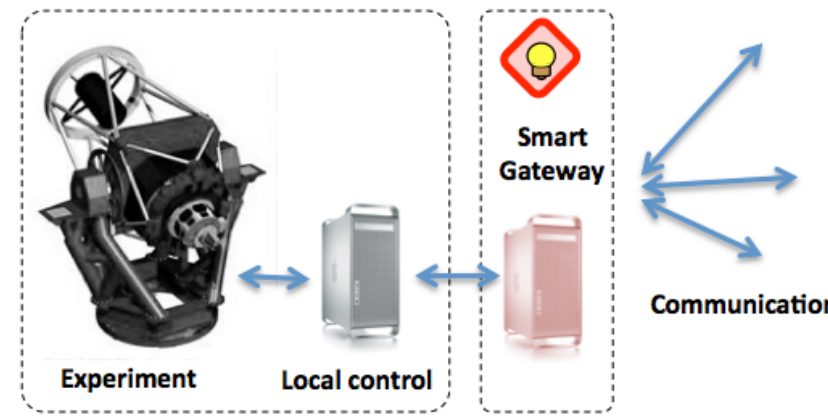

Communication

Figure 3. The Smart Gateway extends existing features.

To federate online labs, smart devices and smart gateways need to efficiently describe their capabilities through metadata. They should also be able to understand other requests [8].

The smart device provides interfaces to its services. While a built-in custom Web server can server static pages/request, it can also serve more dynamic interfaces to its services, this in the form of widgets/gadgets. These widgets can be combined with the help of the App composer and presented to users through the Portal. Widgets aggregations will be save in the Cloud.

If the desired user interface (widget) is not provided by the smart device, an alternative one, designed by another provider can be used instead. This alternative widget can efficiently access smart device raw services via websockets. In addition, many other protocols such as XML/RPC ${ }^{4}$ could also be supported. These smart device interfaces are the smart device APIs, which enable any developer or user to fully and efficiently access the smart device (Fig 4).

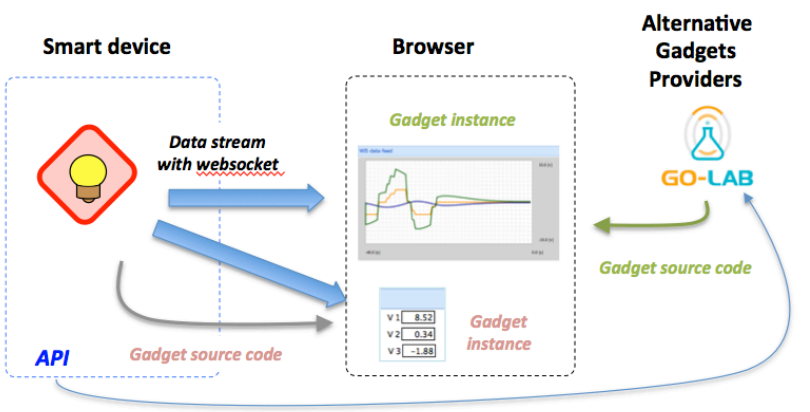

Figure 4. The smart device could provide i) simple gadget, ii) raw data stream via websocket, iii) API for other providers.

The backend infrastructure will also provide services that will allow authentication and the scheduled use of the labs by the users and competences bartering. The learning analytics and other Add-on Services will be developed and exploited by the Go-Lab frontend Portal to provide awareness and adapt the level of scaffolding to students' competences and needs.

The Go-Lab project will define guidelines, standards and best practices for designing and implementing smart devices. It will also develop and extend existing technologies and

\footnotetext{
${ }^{4}$ http://xmlrpc.scripting.com/
} 
standards. The final goal is to define smart devices such that they can simply be plugged in the proposed Cloud services infrastructure and accessed through the Go-Lab Portal.

The Go-Lab Portal (Frontend) will provide an easily accessible platform where users will have the ability to use and personalize their inquiry-learning spaces according to their needs. The Portal will not only include the available labs but also tools and scenarios that will support users in designing their own inquiry based activities. The platform will be compatible with tablets for ubiquitous access.

\section{INQUIRY LEARNING SPACES}

An inquiry learning space is an online space constructed or repurposed by a teacher for a single inquiry learning activity dedicated to students. The teacher view of the space integrates support for its construction, such as recommendation of existing spaces or knowledgeable peers, as well as support for interaction with practice and resources sharing communities. The students' view of the space typically integrates instructions, learning resources, as well as apps supporting the inquiry learning stages (such as orientation, hypothesis, experimentation, and conclusion). For the sake of clarity, if one would compare scientific and social platforms, the proposed portal would correspond to the Facebook or LinkedIn portals, and scaffolded inquiry learning spaces to Facebook profiles or LinkedIn groups; inquiry learning spaces being populated with more advanced resources such as online Labs. Personalization of the inquiry learning spaces is one of the key enabling features of the proposed technical framework.

The example of an inquiry learning space is given in Figure 5. It is supporting the study of the formation of galaxies. Students can use the network of remote telescopes (Faulkes Telescopes ${ }^{5}$ ) to make their own observations of interacting galaxies, they can compare their findings with scientific data from the DSpace scientific repository ${ }^{6}$ and they can use the Galaxy Crash modeling tool ${ }^{7}$ to simulate interactions of galaxies. By changing the parameters of each galaxy (e.g., mass, relative velocity, separation distance, time), students are creating tidal tails and elliptical galaxies, and they are reproducing the types of galaxy interactions, which we observe today. Through the modeling tools students can modify the view of the simulation to see the interactions from different angles to see which best match their observations. Organizing these resources in the framework of a school project or during a normal school lesson is a quite demanding task for teachers. As support, they will get recommendations of inquiry classroom scenarios and will easily rely on their peer communities to share domain- and level-specific practices and solutions.

In the Go-Lab Portal, science teachers will first look at learning spaces created and shared by other colleagues having exploited with their students scenarios related to galaxy collisions in order to illustrate the concept of Gravity. Teachers will then design their own scenario and aggregate resources by repurposing and adapting the best ones (scored by colleagues)

\footnotetext{
5 http://www.faulkes-telescope.com

6 http://www.discoveryspace.net

${ }^{7}$ http://burro.cwru.edu/JavaLab/GalCrashWeb/GCSolo.html
}

to the students' level. To implement a chosen scenario, teachers will create a "Galaxy Collision" space (see Figure 5) in which they will aggregate recommended Web apps for accessing the DSpace scientific repository with preselected images, for providing the Galaxy Crash modeling tool (which will already be available at this time as an OpenSocial widget developed with the use of the App Composer by one of their peers) where they will set meaningful initial conditions for the simulation. They will also add one of the cognitive scaffolding apps proposed by Go-Lab, i.e., a simple experience planner in which students propose some actions and where they will be able to record their trials and add comments. They may also add a hypothesis creation app that helps students create hypotheses. These tools will be presented in a structured inquiry cycle overview.

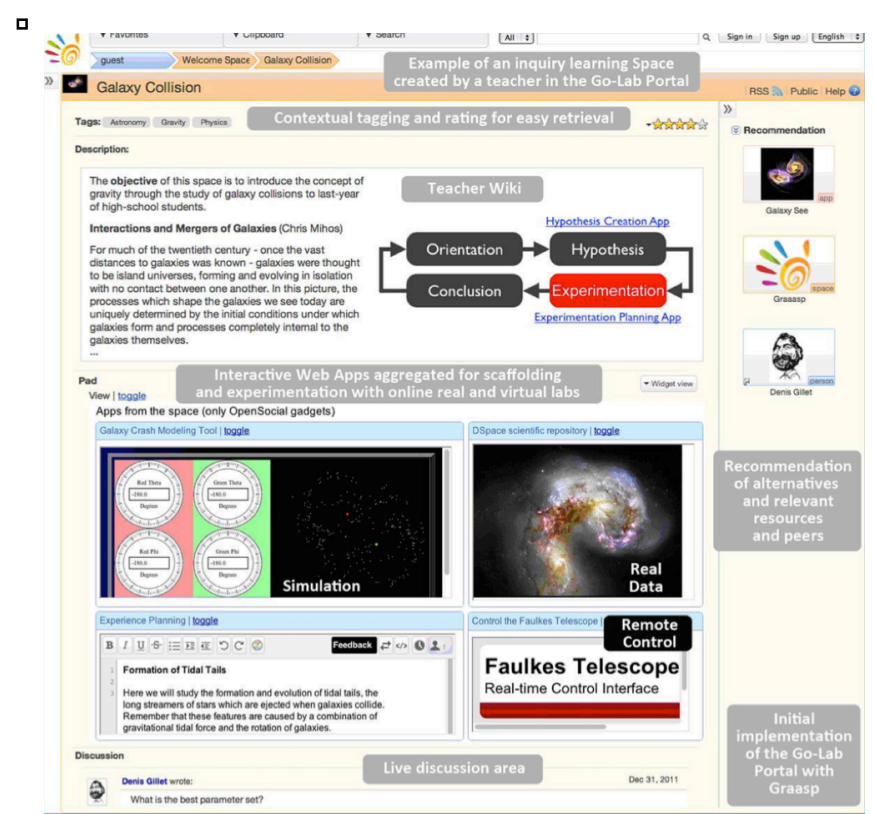

Figure 5. Example of a inquiry learning space in Graasp.

\section{PERSONALIZATION}

Personalization in the STEM Portal relies on a multidimensional approach. The first level of personalization will be handled by teachers at the creation of new or the repurposing of existing inquiry learning spaces. Recommendation of online lab interfacing apps and activity scaffolding apps will be available, as well as targeted search in the federated repositories and in the cloud. The categorization of the resources offered in terms of targeted disciplines (mathematics, physics, environment, ...), big ideas [9], target public levels (primary, lower or upper secondary, ...), inquiry learning skills (novice, expert) and languages, will enable a second level of personalization of both the apps and the spaces. The third level of personalization is a possible reshuffling of the spaces according to the inquiry learning stages as predefined or specified by the teachers, i.e., by providing alternatives apps and supporting resources. As such, this level of personalization is not an adaptation as usually considered in adaptive learning, but an orchestration of the activities and the resources by teachers. The last level of personalization will rely 
on learning analytics, and especially class activity patterns associated with given inquiry-learning spaces. As an example, an experiment design app integrated in a space may inform students on their experimentation behavior through noninvasive hints, but may only do so for inexperienced students. More advanced students may get the recommendation to turn the apps into their expert mode depending on their actual experimentation behavior. The App composer will ease the creation of apps targeting different inquiry learning skill levels and taking advantage of contextual learning analytics, i.e. learning analytics exploiting interaction traces left by students at the level of a given inquiry learning space.

\section{PRIVACY AND USER IDENTITIES}

In a platform dedicated to children, the proper handling of privacy is an essential feature. Privacy is strongly tided to the user identities and login options. To minimize the entry barrier for new or infrequent users, an anonymous access requiring no login will be provided in public inquiry spaces. Such access is available for teachers and children. Obviously, anonymous users will not be tracked and will not get the benefits of personalized scaffolding features. The next level of access with a low entry barrier is tailored for classroom activities. A registered teacher, i.e. any fully identified user, can create an inquiry learning space and issue a single or multiple access token for his or her students. Such tokens (which can take simply the form of shared secret URLs) require no login and enable class patterns to be identified in order to provide the teacher and the students with awareness cues regarding their behaviors and their progresses, and to recommend scaffolding. It will also be possible for a teacher to issue personal tokens, requiring no login for users but enabling an individual supervision or tracking. Only the teacher will know the matching between the token and the name of the corresponding student. Such a scheme is not in contradiction with privacy regulation for children. As a matter of fact, in a traditional classroom, the teacher has such access to personal data and individual behaviors. At any stages, for more advanced personalization and more accurate construction of learning analytics, users can request a fully featured login with a unique ID and a personal password. Inquiry learning spaces may be restricted to such fully identified users or combined with access through tokens. Alternative login with Google or Facebook openIDs is also considered.

\section{FEDERATION OF ONLINE LABS}

Go-Lab starts with a series of online labs from the projects' beneficiaries. These include large science organizations such as ESA and CERN who own online labs on space topics and nuclear physics. Another example is the Faulkes telescope, see the earlier examples in Section IV, that allow students to perform sky observations. In a later stage, online labs from other Go-Lab project partners will be included, for example WebLab-Deusto ${ }^{8}$. In the final stage of the project, online labs from external lab owners will be included.

\section{CONCLUDING REMARKS}

Enabling an easy plug (online and in repositories), play (in learning environments and social media platforms) and share (with peers and communities) of online labs is a challenging task. In this paper, an approach relying on a social media portal enabling the federation of online labs interfaced as smart devices, the interaction within STEM teacher communities, and inquiry learning at school has been presented.

The core inquiry learning enablers are dedicated online spaces provided by teachers and exploited by students for focused activities. Access, privacy enforcement, personalization, scaffolding and learning analytics are tight to these spaces.

\section{ACKNOWLEDGMENT}

The reported work was partially funded by the European Union in the context of the ROLE (Grant Agreement no. 231396) and Go-Lab projects (Grant Agreement no. 317601) under the Information and Communication Technologies (ICT) theme of the 7th Framework Programme for R\&D (FP7) and by the Personal Learning Environment (Phase 3) project of the Swiss AAA/SWITCH Program.

\section{REFERENCES}

[1] T. de Jong, “ Computer simulations - technological advances in inquiry learning”, Science, vol. 312, pp. 532-533, 2006.

[2] D. Gillet, E. Law, ans A. Chatterjee, "Personal learning environments in a global higher engineering education Web 2.0 realm”. In Proceedings of the IEEE Global Engineering Education Conference, 2010.

[3] J. García-Zubia, P. Orduña, I. Angulo, J. Irurzun, and U. Hernández, "Towards a distributed architecture for remote laboratories", International Journal of Online Engineering, vol. 4, pp. 11-14, 2008.

[4] Ch. Salzmann, and D. Gillet, "From online experiments to smart devices", International Journal of Online Engineering, vol. 4, 2008.

[5] Ch. Salzmann, D. Gillet and P. Mullhaupt. "End-to-end adaptation scheme for ubiquitous remote experimentation", in Personal and Ubiquitous Computing, vol. 13, num. 3 / March, p. 181-196, 2009.

[6] Ch. Salzmann, D. Gillet and P. Huguenin. "Remote Experimentation: Improving User Perception Using Augmented Reality". Proceedings of NIWeek 2000 conference, Aug., 2000.

[7] Ch. Salzmann and D. Gillet,., "Challenges in Remote Laboratory Sustainability“, International Conference on Engineering Education, ICEE 2007, Coimbra - Portugal, 3-7 September 2007.

[8] Ch. Salzmann and D. Gillet, "Smart Device Paradigm: Standardization for online labs", Special Track "Standard on Networked Smart Learning Objects for Online Laboratories: IEEE-SA P1876", 4 ${ }^{\text {th }}$ IEEE Global Engineering Education Conference (EDUCON), Berlin, Germany, March 13-15, 2013.

[9] W. Harlen (Ed.), "Principles and big ideas of science education". Hatfield, Herts: Association for Science Education, 2010.

${ }^{8}$ https://www.weblab.deusto.es/web/ 